\title{
A population-based study of copy number variants and regions of homozygosity in healthy Swedish individuals
}

\author{
Shu-Mei Teo ${ }^{1,2,3,5}$, Chee-Seng $\mathrm{Ku}^{2,5}$, Nasheen Naidoo ${ }^{2}$, Per Hall ${ }^{1}$, Kee-Seng Chia ${ }^{1,2,4}$, Agus Salim ${ }^{4}$ \\ and Yudi Pawitan ${ }^{1}$
}

The abundance of copy number variants (CNVs) and regions of homozygosity (ROHs) have been well documented in previous studies. In addition, their roles in complex diseases and traits have since been increasingly appreciated. However, only a limited amount of CNV and ROH data is currently available for the Swedish population. We conducted a population-based study to detect and characterize CNVs and ROHs in 87 randomly selected healthy Swedish individuals using the Affymetrix SNP Array 6.0. More than $600 \mathrm{CNV}$ loci were detected in the population using two different CNV-detection algorithms (PennCNV and Birdsuite). A total of 196 loci were consistently identified by both algorithms, suggesting their reliability. Numerous diseaseassociated and pharmacogenetics-related genes were found to be overlapping with common CNV loci such as CFHR1/R3, LCE3B/3C, UGT2B17 and GSTT1. Correlation analysis between copy number polymorphisms (CNPs) and genome-wide association studies-identified single-nucleotide polymorphisms also indicates the potential roles of several CNPs as causal variants for diseases and traits such as body mass index, Crohn's disease and multiple sclerosis. In addition, we also identified a total of $14815 \mathrm{ROHs} \geqslant 500 \mathrm{~kb}$ or $2814 \mathrm{ROHs} \geqslant 1 \mathrm{M}$ in the Swedish individuals with an average of 170 and 32 regions detected per individual respectively. Approximately $141 \mathrm{Mb}$ or $4.92 \%$ of the genome is homozygous in each individual of the Swedish population. This is the first population-based study to investigate the population characteristics of CNVs and ROHs in the Swedish population. This study found many CNV loci that warrant further investigation, and also highlighted the abundance and importance of investigating ROHs for their associations with complex diseases and traits.

Journal of Human Genetics (2011) 56, 524-533; doi:10.1038/jhg.2011.52; published online 2 June 2011

Keywords: Affymetrix SNP Array 6.0; Birdsuite; copy number variants; PennCNV; regions of homozygosity; Swedish population

\section{INTRODUCTION}

There is a growing body of copy number variant $(\mathrm{CNV})$ maps covering different world populations. ${ }^{1-5}$ Most of these newer studies used high-resolution methods for detecting CNVs, such as the Affymetrix SNP Array 6.0, which has a higher density of singlenucleotide polymorphism (SNP) and copy number probes than previous microarray-based methods. This has led to an improved performance of microarray-based methods to detect smaller CNVs $(<50 \mathrm{~kb}){ }^{1,6}$ In contrast, previous studies have used much lower resolution arrays, such as the bacterial artificial chromosome (BAC) clone or oligonucleotide comparative genomic hybridization arrays and SNP genotyping arrays. ${ }^{7-10}$ Currently, there is only one CNVdetection study in a Swedish population, ${ }^{10}$ but this was performed in a small sample size of 33 individuals and used a low-resolution 32-K bacterial artificial chromosome clone microarray. This has hampered the study from detecting less common and smaller CNVs and from estimating the population frequency of CNVs. The ability to detect smaller CNVs is critical as they are more numerous than the larger CNVs. ${ }^{11}$

In addition, the study by Díaz de Ståhl et al. ${ }^{10}$ was unable to detect regions of homozygosity (ROHs) as the bacterial artificial chromosome clone microarray was unable to generate allelic intensity data. Research on ROHs has started to gain impetus, as evidenced by the increasing number of publications after the first study by Gibson et al. ${ }^{12}$ reported the abundance of $\mathrm{ROH}$ in the human genome of outbred populations. Further studies have investigated the population characteristics of ROHs in healthy individuals, ${ }^{13-15}$ and also performed association analyses to identify ROHs that are associated with complex diseases and traits in a case-control study design. ${ }^{16-18}$

To circumvent the limitations of the previous study by Díaz de Ståhl et al.,$^{10}$ we conducted a study in a Swedish population by genotyping 100 individuals using the Affymetrix SNP Array 6.0 (Affymetrix, Santa Clara, CA, USA). The main aim of this study was to perform a more comprehensive detection of CNVs and $\mathrm{ROHs}$ in the Swedish

${ }^{1}$ Department of Medical Epidemiology and Biostatistics, Karolinska Institutet, Stockholm, Sweden; ${ }^{2}$ Centre for Molecular Epidemiology, Department of Epidemiology and Public Health, Yong Loo Lin School of Medicine, National University of Singapore, Singapore; ${ }^{3}$ NUS Graduate School for Integrative Sciences and Engineering, National University of Singapore, Singapore and ${ }^{4}$ Department of Epidemiology and Public Health, Yong Loo Lin School of Medicine, National University of Singapore, Singapore

5 Joint first author.

Correspondence: C-S Ku, Center for Molecular Epidemiology, National University of Singapore, Singapore 117597, Singapore.

E-mail: csikcs@nus.edu.sg or Professor Y Pawitan, Department of Medical Epidemiology and Biostatistics, Karolinska Institutet, P0 Box 281, 17177 Stockholm, Sweden. E-mail: Yudi.Pawitan@ki.se

Received 18 January 2011; revised 12 April 2011; accepted 25 April 2011; published online 2 June 2011 
population and to describe their population characteristics. Although several studies have been performed to detect and characterize CNVs and $\mathrm{ROHs}$ in multiple European populations, these studies have also documented the genetic differences among these populations. ${ }^{14,15,19}$ The extension of the International HapMap Project to include an additional seven populations in Phase III further suggests that multiple populations from diverse ancestries or different geographical locations are needed to study their population genetics. ${ }^{20}$ These previous studies have justified the need for a population-based study to characterize CNVs and ROHs in healthy Swedish individuals. We also compared the Swedish population with the HapMap phase III populations using principal component analysis.

\section{MATERIALS AND METHODS}

\section{Samples and genotyping platform}

A total of 100 randomly selected healthy Swedish individuals volunteering as controls in case-control studies were studied. Peripheral blood samples of the participants for genomic DNA extraction were drawn and stored at the Karolinska Biobank. Identities of the participants were kept anonymous and no personal identifiers were used. All 100 samples were genotyped using the Affymetrix Genome-Wide Human SNP Array 6.0 as per the manufacturer's protocol. Two samples were removed from further analysis because their genotype call rates were below $98 \%$ and the remaining 98 samples were used for $\mathrm{CNV}$ detection.

\section{CNV-detection algorithms and analyses}

CNV calling using PennCNV. We used two CNV-detection algorithms, namely PennCNV ${ }^{21}$ and Birdsuite, ${ }^{22}$ for both comparison and validation. This study focused only on the CNVs in the 22 autosomes because of the inaccuracy of Birdsuite to detect CNVs in sex chromosomes. Log $R$ ratio and B allele frequency were calculated according to the PennCNV algorithm (http://www. openbioinformatics.org/penncnv/penncnv_tutorial_affygw6.html). Smaller CNVs $(<1 \mathrm{~kb})$ were also included in our analysis, as PennCNV by default does not limit its detection to CNVs $>1 \mathrm{~kb}$ in size. We applied a set of filtering criteria as recommended by the algorithm, namely $\log R$ ratio-s.d $>0.35, \mathrm{~B}$ allele frequency-median $>0.55, \mathrm{~B}$ allele frequency-median $<0.45$ and $\mathrm{B}$ allele frequency-drift $>0.006$ to exclude samples with poor quality of signal intensity data (http://www.openbioinformatics.org/penncnv/). This resulted in a further exclusion of 11 samples, with the final set for analysis consisting of 87 samples. For each sample, PennCNV generated a list of CNVs with their confidence scores. The confidence score is a log Bayes factor that measures the likelihood that the locus harbors an abnormal copy number. A confidence score of $\geqslant 10$ has been recommended as the threshold to classify reliable CNVs. Therefore, we retained all CNVs called with confidence scores $\geqslant 10$ for subsequent analyses. Although the confidence score is only a statistical measure of a true positive, our previous study ${ }^{5}$ found that CNVs with a higher confidence score are more likely to be detected consistently across two genotyping platforms. Therefore, this justifies our decision to retain only reliable CNVs called with a sufficient degree of confidence.

Construction of CNV loci using PennCNV output. The CNVs called by PennCNV were shown to overlap across samples. Thus, we merged or grouped these individual $\mathrm{CNV}$ calls into discrete, non-overlapping loci, with the boundaries of each locus determined by the union of all CNVs that belonged to that particular locus. This construction of CNV loci was needed to estimate the population frequencies and these steps were performed using the methods that we have developed previously. ${ }^{5,23}$ We classified the status of these CNV loci into three categories, 'del' (loci containing deletions), 'dup' (loci containing duplications) and 'del/dup' (loci containing both deletions and duplications).

Copy number polymorphism (CNP) calling using Canary (Birdsuite). Birdsuite software $^{22}$ was also used to analyze the Affymetrix SNP Array 6.0 data. There are two components in the software for detecting copy number changes, namely Canary and Birdseye. Canary was used to determine the integer copy number at each of the predefined 1316 CNPs. The term 'CNPs' used by
McCarroll et al. ${ }^{1}$ is to describe common CNV loci. These 1316 CNPs were found in more than one HapMap II individual and their sizes were also accurately determined. Therefore, we used the Canary component in Birdsuite to determine the integer copy number of the 1316 CNPs in the 87 Swedish samples. These 1316 CNPs are distributed in all the autosomes and sex (X and Y) chromosomes. However, 25 CNPs located in the sex chromosomes were removed because the CNP calling in these chromosomes was less accurate. Thus, the results reported in this study comprised only 1291 CNPs in the 22 autosomes. Confidence statistics generated for the CNPs were also used to identify poor-quality calls, and only integer copy numbers detected with high confidence as recommended by the software (confidence score $>0.1$ ) were used for subsequent analyses.

Correlation analysis of CNPs. We performed a correlation analysis of CNPs and the nearby SNPs. Because the sizes of the CNPs were previously accurately determined by McCarroll et al. ${ }^{1}$ we restricted the analysis to only the CNPs detected by Canary. For each of the 1291 CNPs, SNPs within a 200-kb window from the start and end positions of the CNP were considered. We used the squared Pearson's correlation $\left(r^{2}\right)$ for correlation analysis. The genotype calling of the Affymetrix SNP Array 6.0 was carried out using Birdsuite. In addition, to investigate the potential associations of CNPs with human diseases and traits, the same methods of $r^{2}$ calculations for the 1291 autosomal CNPs and the SNPs that were identified by genome-wide association studies (GWAS) were adopted. The list of GWAS-SNPs was downloaded from the National Human Genome Research Institute website (http://www.genome.gov/gwastudies/) on 26 October 2010.

CNV calling using Birdseye (Birdsuite). In addition to PennCNV, we also used another algorithm, Birdseye, to analyze the same set of data as different algorithms tend to have different sensitivities and specificities for detection of CNVs in different regions throughout the genome. As such, CNV loci detected by PennCNV and Birdseye can be cross-validated. Therefore, we used the Birdseye component in Birdsuite to detect additional CNVs throughout the genome, which was not restricted to the 1316 predefined CNPs. Similarly, only CNVs in autosomal chromosomes were used because of the inaccuracy of Birdseye in the sex chromosomes. CNVs with low confidence, as recommended by the software (confidence score $\leqslant 5$ ), were removed from subsequent analysis.

Construction of CNV loci using Birdseye output. We also constructed CNV loci based on the Birdseye output using methods similar to those applied to the PennCNV output. The cutoff for the confidence score used by PennCNV $(\geqslant 10)$ and Birdseye $(\geqslant 5)$ was recommended by both algorithms. This allowed for greater comparability between the CNV loci detected by these two algorithms.

Comparison of CNV loci detected by PennCNV and Birdsuite. The CNV loci identified by PennCNV and Birdseye were compared as a 'validation' step. We used a 'reciprocal 50\% overlapping' method to compare the CNV loci detected by these two algorithms and considered a CNV locus 'found' by both algorithms when this locus was detected in both PennCNV and Birdseye with an overlap of $\geqslant 50 \%$ of their lengths.

Novel CNV loci. To identify novel CNV loci, we compared the CNV loci detected by PennCNV and Birdseye with the data from the Database of Genomic Variants (DGV). ${ }^{24}$ We used the latest data from the DGV (variation.hg18.v8.aug.2009.txt and indel.hg18.v8.aug.2009.txt) downloaded from the DGV Website (http://projects.tcag.ca/variation/). A CNV locus identified by PennCNV and Birdseye was considered novel if it did not share at least $50 \%$ of its length with any CNV loci cataloged in the DGV. All the downstream analyses after PennCNV and Birdsuite were performed using the statistical software package R (http://www.r-project.org/).

\section{Comparison with HapMap phase III populations}

The CEL files of the Affymetrix SNP Array 6.0 for the seven populations in HapMap phase III project were downloaded from the ftp site (ftp://ftp.ncbi. nlm.nih.gov/hapmap/raw_data/hapmap3_affy6.0/). The HapMap phase III populations studied are people of African ancestry in the southwestern USA (ASW), the Chinese community in Metropolitan Denver, Colorado, USA (CHD), Gujarati Indians in Houston, Texas, USA (GIH), the Luhya in Webuye, 
Kenya (LWK), people of Mexican ancestry in Los Angeles, California, USA (MEX), the Maasai in Kinyawa, Kenya (MKK) and the Tuscans in Italy (TSI). All the samples were analyzed using Canary similarly to the analysis of the Swedish population. Only unrelated samples were included in our study, that is, family-related samples were removed using the 'relationships' file provided by the International HapMap Project. After the sample exclusion step, a total of 594 unrelated samples from the seven HapMap III populations were analyzed: ASW $(n=52)$, CHD $(n=89)$, GIH $(n=89)$, LWK $(n=90)$, MEX $(n=53)$, MKK $(n=132)$ and TSI $(n=89)$. We performed principal component analysis to compare the Swedish population with the HapMap phase III populations using the CNP output generated by Canary.

\section{ROH-detection algorithms and analyses}

In addition to CNVs, we also detected $\mathrm{ROHs}$ using PennCNV in the 22 autosomes of the 87 Swedish individuals. However, we only focused on ROHs $\geqslant 500 \mathrm{~kb}$, as this cutoff was adopted in a previous study. ${ }^{18}$ For each of these we confirmed that they are ROHs by determining the genotypes of the SNPs that fall within each region. We then calculated the percentage of heterozygosity (number of heterozygotes/total number of heterozygotes and homozygotes). We also calculated the percentage of missingness genotypes (number of missingness/total number of SNPs in each ROH). First, we used an arbitrary cutoff of the median of the percentage of heterozygosity $(2.5 \%)$ to allow for some heterozygote calls resulting from calling or genotyping errors. As a result, we removed half of the ROHs with a percentage $>2.5 \%$. Second, we removed $\mathrm{ROHs}$ with $>1 \%$ for the missingness, to remove regions where genotype calling was problematic. Finally, for the remaining $\mathrm{ROHs}$, we also ensured a density of one SNP per $10 \mathrm{~kb}$ to exclude those ROHs that could be spuriously detected by a sparse number of SNPs. As such, for a $500-\mathrm{kb} \mathrm{ROH}$, a minimum of 50 SNPs is required. These three criteria were used as the filters to exclude less reliable ROHs. Several summary statistics were then computed to describe the characteristics of ROHs in the Swedish population.

\section{RESULTS}

\section{Characteristics of CNVs identified by PennCNV}

After filtering unreliable CNV calls, an average of approximately 36 CNVs per individual with a ratio of deletions to duplications of approximately 2.6:1 was discovered (Supplementary Table 1). The number of CNVs per individual ranged from 22 to 65 . The median size of a CNV was $28.6 \mathrm{~kb}$ and approximately $66 \%$ of the CNVs were $<50 \mathrm{~kb}$ and $26 \%$ were $<10 \mathrm{~kb}$ (Supplementary Figure 1). The median size of deletions was approximately fourfold smaller than the median size of duplications.

\section{Characteristics of CNV loci identified by PennCNV}

We merged overlapping CNVs to construct CNV loci and identified 623 loci, of which 476 loci contained deletions ('del-loci'), 102 loci contained duplications ('dup-loci') and 45 loci contained both deletions and duplications ('del/dup-loci'; Table 1). These 623 loci covered approximately $61.52 \mathrm{Mb}$ of the nucleotide sequence and the sum of the lengths for del-loci $(19.83 \mathrm{Mb})$ was smaller than that for duploci $(25.80 \mathrm{Mb})$. Similarly for the individual CNVs (Supplementary Table 1), the average size of del-loci $(41.66 \mathrm{~kb})$ was much smaller than that of dup-loci $(252.93 \mathrm{~kb}$; Table 1). More than $77 \%$ of the del-loci were $<50 \mathrm{~kb}$, and in comparison only $22.55 \%$ of dup-loci were within this size range. The majority $(62.75 \%)$ of dup-loci ranged from 50 to $500 \mathrm{~kb}$. In summary, there were far more del-loci, but their sizes tended to be smaller than those of dup-loci. A list of the 623 loci is shown in Supplementary Table 2.

Of the $623 \mathrm{CNV}$ loci, 268 loci were detected in $\geqslant 2$ individuals (Table 1). The remaining loci were detected in only one individual; these loci were not necessarily 'singleton loci' as we only studied

Table 1 Summary statistics of CNV loci constructed from PennCNV output

\begin{tabular}{|c|c|c|c|}
\hline Summary statistics of CNV loci (PennCNV output) & Total & Del & Dup \\
\hline Number of CNV loci & 623 & $476(76.40 \%)^{a}$ & $102(16.37 \%)^{\mathrm{a}}$ \\
\hline Number of CNV loci detected in $\geqslant 2$ individuals & $268(43.02 \%)^{b}$ & $194(40.76 \%)^{b}$ & $29(28.43 \%)^{b}$ \\
\hline Sum of the length of loci (Mb) & 61.52 & 19.83 & 25.80 \\
\hline Average length per locus (kb) & 98.75 & 41.66 & 252.93 \\
\hline Average number of markers per locus & 58 & 34 & 141 \\
\hline \multicolumn{4}{|l|}{ Size distribution } \\
\hline$<10 \mathrm{~kb}$ & $141(22.63 \%)$ & $132(27.73 \%)$ & $6(5.88 \%)$ \\
\hline$\geqslant 10-<50 \mathrm{~kb}$ & $265(42.54 \%)$ & $236(49.58 \%)$ & $17(16.67 \%)$ \\
\hline$\geqslant 50-<100 \mathrm{~kb}$ & $79(12.68 \%)$ & $54(11.34 \%)$ & $21(20.59 \%)$ \\
\hline$\geqslant 100-<500 \mathrm{~kb}$ & $110(17.66 \%)$ & $52(10.92 \%)$ & $43(42.16 \%)$ \\
\hline$\geqslant 500 \mathrm{~kb}$ & $28(4.49 \%)$ & $2(0.42 \%)$ & $15(14.71 \%)$ \\
\hline \multicolumn{4}{|l|}{ Overlapping with DGV } \\
\hline CNV loci that overlap & $388(62.28 \%)$ & $298(62.61 \%)$ & $54(52.94 \%)$ \\
\hline CNV loci that did not overlap & $235(37.72 \%)$ & $178(37.39 \%)$ & $48(47.06 \%)$ \\
\hline \multicolumn{4}{|l|}{ Overlapping with UCSC genes } \\
\hline CNV loci that overlap & $202(32.42 \%)$ & $135(28.36 \%)$ & $51(50.00 \%)$ \\
\hline CNV loci that did not overlap & $421(67.58 \%)$ & $341(71.64 \%)$ & $51(50.00 \%)$ \\
\hline \multicolumn{4}{|c|}{ Overlapping with CNV loci from Birdseye data and consistent in CNV status that is, del/dup/del+dup } \\
\hline CNV loci that overlap & $196(31.46 \%)$ & $160(33.61 \%)$ & $30(29.41 \%)$ \\
\hline CNV loci that did not overlap & $427(68.54 \%)$ & $316(66.39 \%)$ & $72(70.59 \%)$ \\
\hline
\end{tabular}

Abbreviations: CNV, copy number variant; DGV, database of genomic variants; UCSC, University of California Santa Cruz genes.

aThe percentage was calculated by dividing 623 loci.

bThe percentage was calculated by dividing 623,476 and 102 loci, respectively.

Note: As there are only 45 CNV loci $(7.22 \%)$ with status del+dup, the summary statistics of these loci are not shown in the table. A full colour version of this Table is available at the Journal of Human Genetics Journal online. 
87 individuals. The proportion of del-loci detected in $\geqslant 2$ individuals $(40.76 \%)$ was much higher than the proportion for dup-loci $(28.43 \%)$. Among the high-frequency CNV loci (loci that were detected in multiple individuals), several overlapped with disease-related genes such as WWOX and ERBB4 (gastric and pancreatic cancers and melanoma $)^{25-27}$ and CACNA1C (bipolar disorder) ${ }^{28}$ or drug-metabolizing genes such as GSTT1 $^{29}$ (Supplementary Table 2). For example, a deletion locus overlapping with WWOX (a tumor suppressor gene) was detected in 24 of the 87 individuals (27.6\%), and a deletion locus encompassing GSTT1 was deleted at a population frequency of $13.8 \%$. In addition, the proportion of del-loci encompassing the UCSC genes $(28.36 \%)$ was much lower than dup-loci $(50.00 \%)$ overall.

Detection of CNVs using microarrays is usually plagued with poor specificity or a high false-positive rate. In an effort to validate the 623 CNV loci constructed from the PennCNV output, we compared them with the CNV loci detected by Birdseye. We found 196 loci (31.46\%) with $\geqslant 50 \%$ reciprocal overlap with the Birdseye data and the status of 'del', 'dup' and 'del/dup' of the 196 loci were consistent with the Birdseye data. For the remaining $427 \mathrm{CNV}$ loci that were not confirmed by Birdseye data, we found that 247 loci had been cataloged in the DGV (please see Materials and methods). Therefore, by applying two different ways of validation, $443(71.1 \%)$ of the 623 $\mathrm{CNV}$ loci detected by PennCNV were considered reliable in this study (Table 1).

\section{Characteristics of CNPs identified by Canary (Birdsuite)}

Approximately $49.81 \%$ of the 1291 autosomal CNPs were nonpolymorphic in the Swedish population (Supplementary Table 3). The population frequency distribution pattern of the 1291 CNPs is shown in Supplementary Figure 2. Among the polymorphic loci (648 CNPs) and non-polymorphic CNPs (643 loci) in the Swedish population, 289 loci $(44.60 \%)$ and 255 loci $(39.66 \%)$ overlapped with genes or entries from the UCSC annotation of the human genome, respectively. No substantial difference was observed between the polymorphic and non-polymorphic loci.

The majority of the 648 polymorphic CNPs were biallelic (545 CNPs or $84.1 \%$ ), of which the integer copy numbers were either exclusively deletions, that is, copy number of 0 or 1 (387 CNPs or $59.7 \%$ ), or exclusively duplications, that is, copy number of 3 or 4 (158 CNPs or $24.4 \%)$. Among the biallelic $545 \mathrm{CNPs}$, only one showed significant deviation from HWE at an FDR $<0.01$.

Numerous CNPs were found to overlap with important known disease- or pharmacogenetics-related genes (Table 2). The frequencies of these CNPs ranged from relatively uncommon (2.78\% for CNP118) to completely polymorphic (100\% for CNP88). For example, CNP88 overlapped with GSTM1 and GSTM2 was found to be completely deleted in the Swedish population, where all except one carried twocopy deletions. However, it is noteworthy that in approximately half of the sample (47 individuals), the integer copy numbers were successfully determined with high confidence scores. In addition, high deletion frequencies were also found for CNPs overlapping with other GST enzymes such as GSTT1 (60.00\%), GSTT2, GSTT2B and GSTTP1 (98.65\%). Two-copy deletion was common for these enzymes- $17.6 \%$ of the individuals for GSTT1 (CNP2560) and $43.2 \%$ for the other GST enzymes (CNP2559).

Besides these phase II metabolizing enzymes, several disease-associated genes were also found to overlap with these CNPs, such as the FCG receptor genes (autoimmune or inflammatory diseases), ${ }^{30}$ $T P 63^{31}$ and $W W O X^{26}$ (lung adenocarcinoma, gastric, pancreatic and other cancers), CFHR3 and CFHR1 (age-related macular degeneration), ${ }^{32}$ UGT2B17 (prostate cancer and graft-versus-host disease), ${ }^{33,34}$

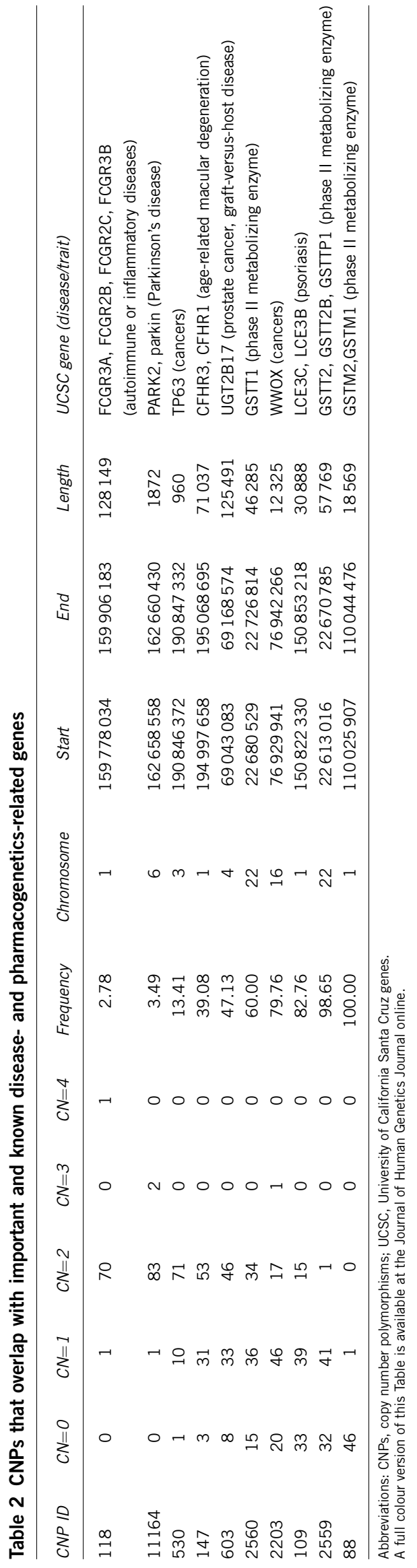

Journal of Human Genetics 

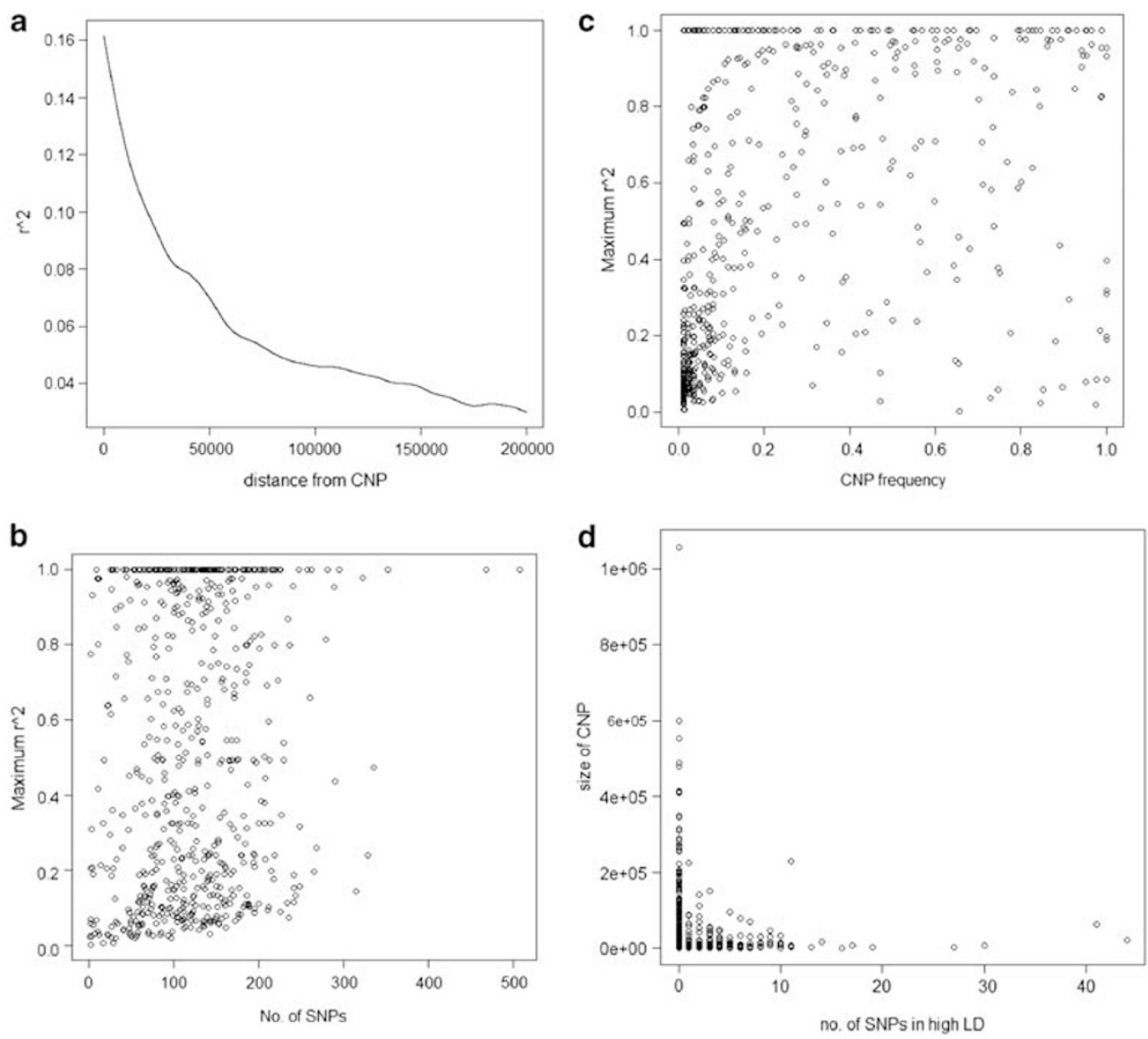

Figure 1 (a) The correlation between the $r^{2}$ and the distance between copy number polymorphism (CNP) and single-nucleotide polymorphism (SNP). (b) Maximum $r^{2}$ of CNP versus number of nearby SNPs in 200-kb windows. (c) Maximum $r^{2}$ of CNP versus CNP frequency. (d) Number of SNPs in strong correlation with the size of CNPs.

and $L C E 3 C$ and $L C E 3 B$ (psoriasis and rheumatoid arthritis) among others. $^{35,36}$ The high deletion frequency of loci overlapping with LCE3C and LCE3B (82.76\%), UGT2B17 (47.13\%) and WWOX $(79.76 \%)$ requires further studies to investigate their associations with complex diseases such as psoriasis, rheumatoid arthritis and graftversus-host disease for hematopoietic stem cell transplantation patients. For example, the mismatch of the copy numbers of UGT2B17 was found to be associated with graft-versus-host disease in patients with hematopoietic stem cell transplantation. ${ }^{34}$ Deletion of UGT2B17 was also associated with an increased risk for prostate cancer. ${ }^{33}$

\section{Correlation analyses between CNPs and nearby SNPs}

To study the correlation patterns with SNPs, we calculated the $r^{2}$ between the 648 polymorphic CNPs and nearby SNPs within a 200-kb window from the start and end positions of the CNP. The proportion of the CNPs with at least one SNP in strong correlation $\left(r^{2}>0.8\right)$ was $31.9 \%$, that is, $207 \mathrm{CNPs}$ were found to be in strong correlation with at least one SNP. The median and maximum numbers of SNPs that were in strong correlation with the $207 \mathrm{CNPs}$ were 3 and 44, respectively. This suggests that half of the $207 \mathrm{CNPs}$ can be tagged by more than three SNPs and some of the CNPs were tagged by tens of SNPs. These results suggest that the majority of CNPs were not being well tagged by the nearby SNPs in the Affymetrix SNP Array 6.0. The strength of the $r^{2}$ value decreases with distance between the CNP and SNP (Figure 1a). We further investigated whether CNPs that were not well tagged tend to be located in the genomic regions where
SNP markers are sparse. The correlation patterns do not appear to be affected by the number of nearby SNPs and the frequencies of CNPs (Figures $1 \mathrm{~b}$ and $\mathrm{c}$ ). In other words, there was no apparent difference in the number of nearby SNPs and the frequencies of CNPs between (a) the CNPs that were in strong correlation $\left(r^{2}>0.8\right)$ and (b) CNPs that were not in strong correlation with SNPs (Figures $1 b$ and $c$ ). However, smaller-sized CNPs were generally in strong correlation with more SNPs than the larger CNPs (Figure 1d).

\section{Correlation analyses between CNPs and GWAS-SNPs}

To investigate the potential role of CNPs in the etiology of complex diseases or traits, we computed the $r^{2}$ between CNPs and the SNPs on the NHGRI GWAS Catalog (http://www.genome.gov/gwastudies/). Of the $>3000$ GWAS-SNPs that have been found to be associated with various complex diseases and traits, only eight GWAS-SNPs were found to be in strong correlation with six CNPs (Table 3). Following the methods of Conrad et al., ${ }^{2}$ we define in our analysis a strong correlation as $r^{2}>0.5$. These eight SNPs were reported to be associated with five diseases or traits, namely body mass index, childhood acute lymphoblastic leukemia, early-onset myocardial infarction, Crohn's disease and multiple sclerosis. Several SNPs were in strong correlation with a single CNP, for example, three SNPs (rs13361189, rs1000113 and rs11747270) were found to be in strong correlation with CNP874.

The most notable SNP was rs2815752 near the NEGR1 gene (associated with body mass index), which was in perfect correlation $\left(r^{2}=1\right)$ with CNP60. This locus is a $42-\mathrm{kb}$ deletion located in 
Table 3 Correlation between CNPs and GWAS-SNPs at $r^{2}>0.5$

\begin{tabular}{|c|c|c|c|c|c|c|c|c|}
\hline$C N P I D$ & Chromosome & Start position & End position & Length & GWAS-SNP & $r^{2}$ value & Gene & Complex disease/trait \\
\hline 60 & 1 & 72541504 & 72583736 & 42232 & rs2815752 & 1 & NEGR1 & BMI \\
\hline 147 & 1 & 194997658 & 195068695 & 71037 & rs6428370 & 0.647399825 & Intergenic & Acute lymphoblastic leukemia (childhood) \\
\hline 333 & 2 & 203608045 & 203610291 & 2246 & rs6725887 & 0.84632626 & WDR12 & Myocardial infarction (early onset) \\
\hline 874 & 5 & 150185693 & 150198797 & 13104 & rs13361189 & 0.927251567 & IRGM & Crohn's disease \\
\hline 874 & 5 & 150185693 & 150198797 & 13104 & rs1000113 & 0.927251567 & IRGM & Crohn's disease \\
\hline 874 & 5 & 150185693 & 150198797 & 13104 & rs11747270 & 0.927251567 & IRGM & Crohn's disease \\
\hline 877 & 5 & 155409350 & 155415307 & 5957 & rs4704970 & 1 & SGCD & Multiple sclerosis \\
\hline 933 & 6 & 32539530 & 32681749 & 142219 & rs3129934 & 0.664781909 & HLA-DRB1 & Multiple sclerosis \\
\hline
\end{tabular}

Abbreviations: BMI, body mass index; CNPs, copy number polymorphisms; GWAS, genome-wide association studies; SNP, single-nucleotide polymorphism.

A full colour version of this Table is available at the Journal of Human Genetics Journal online.

chromosome 1 that did not overlap with any of the UCSC genes and is located only $1.3 \mathrm{~kb}$ away from the SNP. The total deletion frequency in the Swedish population was high (Table 3 and Supplementary Table 4 ), of which $51.72 \%$ were one-copy deletions and $29.89 \%$ were twocopy deletions. CNP874 was found to be in nearly perfect correlation $\left(r^{2}=0.93\right)$ with three GWAS-SNPs located near the IRGM gene, which is associated with Crohn's disease. However, in comparison with CNP60, the total deletion frequency for CNP874 was much lower, with only $11.90 \%$ one-copy deletions and $1.19 \%$ two-copy deletions. This locus spans $13 \mathrm{~kb}$ in chromosome 5 and does not overlap with any of the UCSC genes. The three GWAS-SNPs were located $4.8 \mathrm{~kb}$ (rs13361189), $21.4 \mathrm{~kb}$ (rs1000113) and $40.2 \mathrm{~kb}$ (rs11747270) away from the deletion. The CNP877 locus is implicated in multiple sclerosis, where it is in perfect correlation with the GWAS-SNP (rs4704970). None of the individuals were deleted in both copies, and $32.56 \%$ were one-copy deletions. The other CNPs were implicated in childhood acute lymphoblastic leukemia (CNP147) and early-onset myocardial infarction (CNP333). Interestingly, all the CNPs found to be in strong correlation with GWAS-SNPs had only deletions in the loci.

\section{Characteristics of CNV loci identified by Birdseye (Birdsuite)}

Similar to the PennCNV output analysis, we also merged overlapping CNVs to construct CNV loci for the Birdseye data and identified 641 loci, of which 451 were del-loci, 102 were dup-loci and the remaining 31 were del/dup-loci (Table 4). The proportion of del-loci $(76.40 \%)$ identified by PennCNV data was higher than that for the Birdseye data $(70.36 \%)$. In comparison, the Birdseye data identified a higher proportion of dup-loci $(24.80 \%)$ than the PennCNV data (16.37\%). However, these differences are not substantial.

The 641 loci identified by the Birdseye data cover approximately $35.23 \mathrm{Mb}$ of the nucleotide sequence, and the sum of the length for del-loci $(13.10 \mathrm{Mb})$ is smaller than that for dup-loci $(15.06 \mathrm{Mb})$. Similar to PennCNV data, the average size of del-loci $(29.04 \mathrm{~kb})$ is much smaller than that of the dup-loci $(94.70 \mathrm{~kb})$. However, substantial differences were observed for these parameters between the PennCNV and Birdseye data (Tables 1 and 4). For example, the sum of lengths covering CNV loci detected by the PennCNV data $(61.52 \mathrm{Mb})$ was approximately twice that for the Birdseye data (35.23 Mb), while they have an almost similar number of CNV loci.

More than $60 \%$ of del-loci were $<10 \mathrm{~kb}$, and in comparison, only $18.24 \%$ of dup-loci fall within this size range. The majority $(52.20 \%)$ of dup-loci ranged from 10 to $100 \mathrm{~kb}$. In summary, there were more del-loci, but their sizes tended to be smaller than those of the dup-loci. This is in agreement with the PennCNV data. However, the size distribution pattern of the $\mathrm{CNV}$ loci for the Birdseye data is skewed towards the 'smaller' end compared with the PennCNV data. This is apparent when comparing the proportions in the first two strata: (a) $<10 \mathrm{~kb}$ and (b) $\geqslant 10-<50 \mathrm{~kb}$ between the two sets of data (Tables 1 and 4). The list of the 641 loci is shown in Supplementary Table 2.

Of the $641 \mathrm{CNV}$ loci, 280 loci were detected in $\geqslant 2$ individuals (Table 4), and the remaining loci in only one individual. The proportion of del-loci detected in $\geqslant 2$ individuals $(43.90 \%)$ was much higher than the proportion for dup-loci (32.08\%). Among the high-frequency CNV loci (loci detected in multiple individuals), several overlapped with disease-associated or pharmacogeneticsrelated genes such as WWOX and GSTT1, which have also been observed in the PennCNV data (Supplementary Table 2). Furthermore, the deletion frequencies were comparable between the Birdseye and PennCNV data. For example, a deletion locus overlapped with WWOX was also found in the Birdseye data. It was detected in 29 of the 87 individuals (33.33\%), and a deletion locus encompassing GSTT1 was deleted at a population frequency of $11.49 \%$. Among the $196 \mathrm{CNV}$ loci (160 del-loci, 30 dup-loci and $6 \mathrm{del} / \mathrm{dup}-$ loci) that were detected by both the Birdseye and PennCNV data and consistent in their CNV status, only 21 loci differed significantly (FDR <0.01) in their frequencies estimated by both sets of data. In addition, the proportion of del-loci encompassing UCSC genes (24.83\%) was much lower than dup-loci (45.28\%); this finding is again consistent with the PennCNV data.

For the CNV loci detected with the Birdseye data, we also performed the 'validation' steps for overlap with the PennCNV data and the DGV. As mentioned earlier, we found 196 loci with $\geqslant 50 \%$ reciprocal overlap between the Birdseye and PennCNV data. For the remaining $445 \mathrm{CNV}$ loci that were not confirmed by PennCNV data, we found that 322 loci have been cataloged in the DGV (please see Materials and methods). Therefore, by applying two different ways of validation, $518(80.81 \%)$ of the $641 \mathrm{CNV}$ loci detected by Birdseye were considered reliable in this study (Table 4 ).

\section{Comparison with HapMap phase III populations}

The principal component analysis showed distinct clusters for populations with different ancestries. The first two principal components (PC1 and PC2) separated the African (ASW, MKK and LWK) and non-African (CHD, GIH, MEX, SWED and TSI) populations (Figure 2a). This suggests that the CNP profiles of the African populations were substantially different from those of the non-African populations. From the second and fourth principal components (PC2 and PC4), three distinct clusters were observed (Figure 2b). The three African populations remained as a distinct cluster; however, CHD was separated from the European populations (MEX, SWED and TSI) and the Gujarati Indians (GIH). This indicates that the CNP profile of Gujarati Indians in Houston (Texas, USA) resembles that of the European populations. Principal component analysis was also performed by restricting only the 'European cluster' populations 
Table 4 Summary statistics of CNV loci constructed from Birdseye (Birdsuite) output

\begin{tabular}{|c|c|c|c|}
\hline Summary statistics of CNV loci (Birdseye output) & Total & Del & Dup \\
\hline Number of CNV loci detected in $\geqslant 2$ individuals & $280(43.68 \%)^{b}$ & $198(43.90 \%)^{b}$ & $51(32.08 \%)^{\mathrm{b}}$ \\
\hline Average length per locus & $54.96 \mathrm{~kb}$ & $29.04 \mathrm{~kb}$ & $94.70 \mathrm{~kb}$ \\
\hline Average number of markers per locus & 30 & 22 & 42 \\
\hline \multicolumn{4}{|l|}{ Size distribution } \\
\hline$\geqslant 10-<50 \mathrm{~kb}$ & $193(30.11 \%)$ & $119(26.39 \%)$ & $63(39.62 \%)$ \\
\hline$\geqslant 50-<100 \mathrm{~kb}$ & $52(8.11 \%)$ & 27 (5.99\%) & $20(12.58 \%)$ \\
\hline$\geqslant 100-<500 \mathrm{~kb}$ & $79(12.32 \%)$ & $31(6.87 \%)$ & $40(25.16 \%)$ \\
\hline$\geqslant 500 \mathrm{~kb}$ & $14(2.18 \%)$ & $2(0.44 \%)$ & $7(4.40 \%)$ \\
\hline \multicolumn{4}{|l|}{ Overlapping with DGV } \\
\hline \multicolumn{4}{|l|}{ Overlapping with UCSC genes } \\
\hline CNV loci that overlap & $202(31.51 \%)$ & $112(24.83 \%)$ & $72(45.28 \%)$ \\
\hline CNV loci that did not overlap & $439(68.49 \%)$ & $339(75.17 \%)$ & $87(54.72 \%)$ \\
\hline \multicolumn{4}{|c|}{ Overlapping with CNV loci constructed from Birdseye and consistent in CNV status, that is, del/dup/del+dup } \\
\hline CNV loci that overlap & $196(30.58 \%)$ & $160(35.48 \%)$ & $30(18.87 \%)$ \\
\hline CNV loci that did not overlap & $445(69.42 \%)$ & $291(64.52 \%)$ & $129(81.13 \%)$ \\
\hline
\end{tabular}

Abbreviations: CNV, copy number variant; DGV, database of genomic variants; UCSC, University of California Santa Cruz genes.

aThe percentage was calculated by dividing 641 loci.

bThe percentage was calculated by dividing 641,451 and 159 loci, respectively.

Note: as there are only $31 \mathrm{CNV}$ loci $(4.84 \%)$ with status del+dup, the summary statistics of these loci were not shown in the table.

A full colour version of this Table is available at the Journal of Human Genetics Journal online.

(GIH, MEX, SWED and TSI) in PC2 versus PC4 (Figure 2b). More interestingly, we also found that the CNP profile of the Swedish population was substantially different from that of the other populations such as GIH and MEX, but it was also appreciably different from that of TSI (Figure 2c). These differences further justify the need to detect and characterize the CNV/CNP profile of the Swedish population.

\section{Characteristics of ROHs}

By restricting ROHs to $\geqslant 500 \mathrm{~kb}$, a total of 14815 regions were found in the 87 Swedish individuals with an average of 170 ROHs (Supplementary Table 5). The number of ROHs ranged from 105 to 220. The majority of these ROHs were $<1 \mathrm{Mb}$ in length (Supplementary Figure 3 ). However, by restricting $\mathrm{ROHs}$ to $\geqslant 1 \mathrm{Mb}, 2814 \mathrm{ROHs}$ with an average of $32 \mathrm{ROHs}$ per individual were found. The median size of the ROHs was approximately $686 \mathrm{~kb}$, with the largest $\mathrm{ROH}$ spanning a length of approximately $25 \mathrm{Mb}$ in chromosome 11 . This $\mathrm{ROH}$ contained 9034 homozygotes, 29 heterozygotes and 2 missing genotypes, and had a density of $3.6 \mathrm{SNPs}$ per $10 \mathrm{~kb}$. The second largest ROH was $12 \mathrm{Mb}$ in length and was detected in a different individual. This $\mathrm{ROH}$ contained 1571 homozygotes and 19 heterozygotes and had a density of 1.3 SNPs per $10 \mathrm{~kb}$. The sum of the length of ROHs in each individual (that is, the total length of all the $\mathrm{ROHs}$ in one individual) was then computed. It ranged from approximately 87 to $179 \mathrm{Mb}$ with a median and mean of approximately $141 \mathrm{Mb}$, respectively. This finding suggests that, on average, $141 \mathrm{Mb}$ or $4.92 \%$ of the human genome $(2867 \mathrm{Mb})$ was homozygous in these Swedish individuals (Table 5).

The distribution pattern of these ROHs in the 22 autosomes was also studied. The larger chromosomes (chromosomes 1-8) tended to have a higher average number of ROHs per individual (Table 5). For example, these chromosomes had an average number of $>9$ ROHs per individual, and in contrast, an average number of $<5$ ROHs per individual was detected in chromosomes 16-22. As a result, chromosomes 1-8 also had a higher average sum of length of ROHs per individual $(>7 \mathrm{Mb})$ than the smaller chromosomes, that is, $<4 \mathrm{Mb}$ for chromosomes $16-22$. However, this pattern was less obvious when the parameters were adjusted for the sizes of the chromosomes. For example, the proportion of the chromosome encompassed by $\mathrm{ROH}$ for the largest chromosome 1 (4.78\%) was smaller than that for the other chromosomes such as chromosome 17 (5.14\%). An apparent trend is not observed for the proportion of the chromosome encompassed by ROHs across the 22 autosomes. However, chromosomes 3, 4, 8 and 12 tended to have the highest proportions (5.90-6.16\%), and, in contrast, chromosomes 16, 19, 21 and 22 had the lowest proportions (1.76-2.59\%). These results were not due to differences in the density of SNPs across the 22 autosomes, as we found no substantial differences in the density of SNPs across the chromosomes (except for chromosome 19, which had a density of $<2$ SNPs per $10 \mathrm{~kb}$ when compared with the other chromosomes). Although chromosomes 3 and 4 had $>6 \%$ of the proportion of the chromosome encompassed by ROHs, the density of SNPs of these chromosomes was similar to that of chromosome 16, where only approximately $2 \%$ of this chromosome was covered by ROHs (Table 5).

\section{DISCUSSION}

In this study, > $600 \mathrm{CNV}$ loci were detected in the Swedish population using two different CNV-detection algorithms, that is, PennCNV (623 loci) and Birdsuite (641 loci). From these, 196 loci were consistently identified by both algorithms, suggesting their reliability. In addition, 

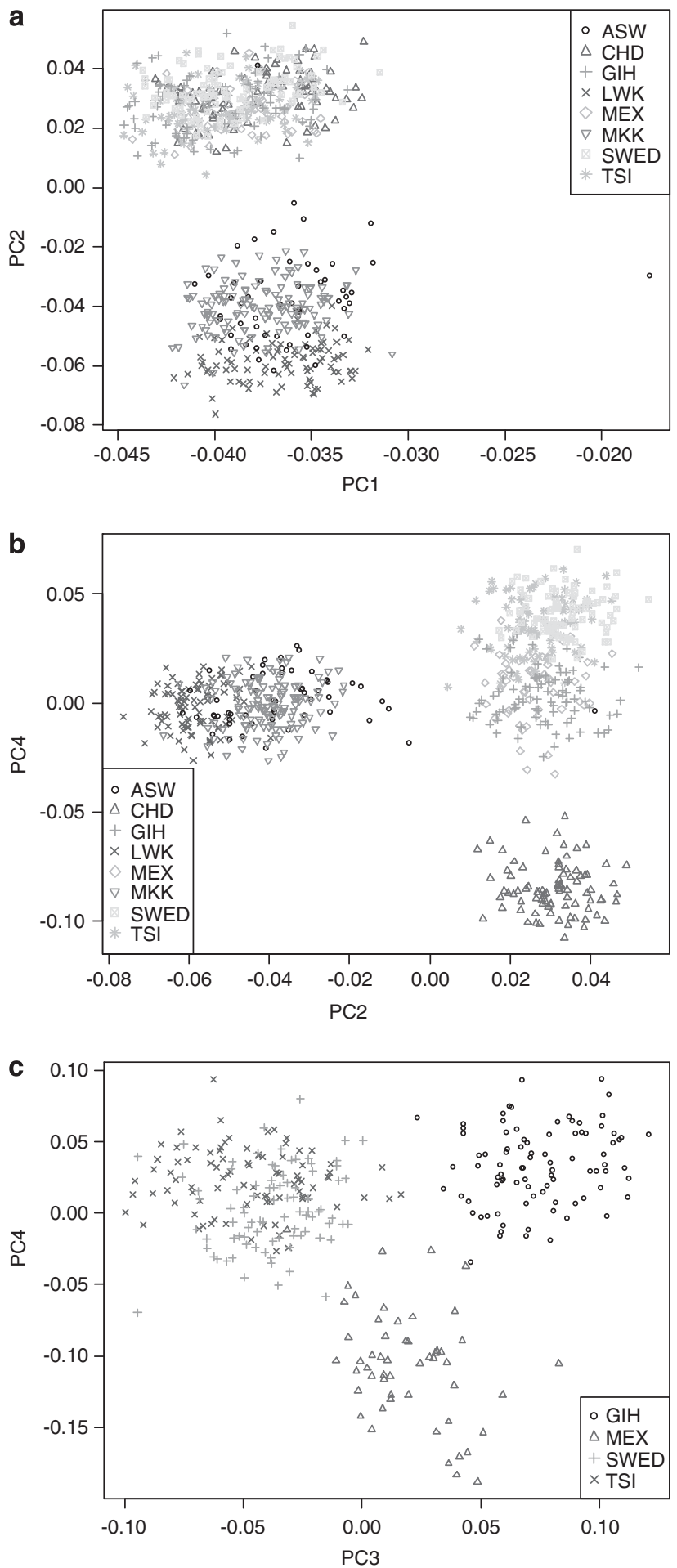

Figure 2 Principal component analysis comparing the populations. (a) Swedish and HapMap phase III populations-PC 1 versus PC 2. (b) Swedish and HapMap phase III populations-PC 2 versus PC 4. (c) Swedish and three HapMap III populations (GIH, MEX and TSI)_PC 3 versus PC 4.

we also identified a total of $14815 \mathrm{ROHs} \geqslant 500 \mathrm{~kb}$ or $2814 \mathrm{ROHs}$ $\geqslant 1 \mathrm{Mb}$ in the Swedish individuals with an average of 170 and 32 regions detected per individual, respectively.

$\mathrm{CNV}$ s have been increasingly recognized as a significant source of genetic variation or diversity in human populations. Detection of
CNVs using SNP genotyping arrays is more cost-effective and affordable for population-based studies as compared with sequencing-based methods, which are limited to only a few individuals. ${ }^{37-39}$ This has enabled our study to investigate the population characteristics of CNVs. Although $>600 \mathrm{CNV}$ loci were identified, only 268 were detected in at least two individuals by PennCNV. Similarly, Birdseye also found 280 common CNV loci in the 87 Swedish individuals. More importantly, these common CNV loci were found to encompass several disease-related and important drug-metabolizing genes, suggesting that these loci warrant further characterization and study for their associations with the relevant diseases or traits.

We applied two different algorithms to detect CNV loci as a validation step; 196 loci were found by both the algorithms and these loci were also consistent in their CNV status ('del', 'dup' or 'del+dup'). In the majority of the 196 loci, the population frequencies were also in good agreement between PennCNV and Birdseye data, indicating that these $\mathrm{CNV}$ loci are highly reliable. In addition, most of the CNV loci detected by PennCNV ( $>70 \%)$ and Birdseye $(>80 \%)$ can be 'validated' by comparing them with each other and with the DGV. The proportion of CNV loci overlapping with the DGV was approximately $62 \%$ and $72 \%$ for PennCNV and Birdsuite, respectively. These percentages could be overestimated because of the false-positive entries in the DGV. Of the $196 \mathrm{CNV}$ loci that were identified by both algorithms, 53 loci had not been previously cataloged in the DGV, which represents a subset of reliable novel CNV loci identified in our study. The list of CNV loci in the DGV is not as yet complete as results from only 42 published studies were documented as of November 2010 (http://projects.tcag.ca/variation/).

On performing the correlation analysis between CNPs and GWAS-SNPs, our results also indicated that several CNPs could be potential causal variants because of their strong correlation with the GWAS-SNPs. Notably, the strong correlation between the CNPs and the GWAS-SNPs near NEGR1 and IRGM for body mass index and Crohn's disease, respectively, are consistent with previous studies. ${ }^{40,41}$

Our study has a higher sensitivity than the study by Díaz de Ståhl et al., ${ }^{10}$ which only detected an average of $15 \mathrm{CNVs}$ per individual compared with our study, which detected an average of $36 \mathrm{CNVs}$ per individual. An average of 4 clones per CNV was detected in the Díaz de Ståhl et al. study, whereas in our study, each CNV was detected by an average of 51 markers (Supplementary Table 1). The ability to detect smaller CNVs was also demonstrated in our study, because the average size of CNVs detected by Díaz de Ståhl et al. was approximately 3.5 -fold $(358 \mathrm{~kb}$ ) larger than that in our study. Although Díaz de Ståhl et al. also clustered individual overlapping CNVs into loci, their analysis was performed using data from different ancestries (33 Europeans, 24 Africans and 14 Asians), whereas the CNV loci constructed in our study were based entirely on the data from 87 Swedish individuals. Therefore, our list of CNV loci and their frequencies was more representative of the Swedish population.

We did not compare our results with existing data from published studies because of the methodological issues in $\mathrm{CNV}$ and $\mathrm{ROH}$ detection in the different studies. As different studies have used different platforms, quality control criteria and methods to construct $\mathrm{CNV}$ loci and detect ROHs, comparisons with published studies would not be valid. Therefore, we would need to analyze the data from different populations with same analytical procedure. Furthermore, such a comparison is beyond the scope of the current paper and will be addressed in a future publication. However, to provide some preliminary insight into the population differences, we compared the CNP profiles of the Swedish population with the HapMap phase III populations. This comparison was appropriate as 
Table 5 Distribution pattern of ROHs across the 22 autosomes

\begin{tabular}{|c|c|c|c|c|c|c|c|c|}
\hline Chromosome & $\begin{array}{c}\text { Total number } \\
\text { of } \mathrm{ROHs}\end{array}$ & $\begin{array}{l}\text { Average number } \\
\text { of } R O H s \text { per individual }\end{array}$ & $\begin{array}{l}\text { Sum of length } \\
\text { of } \mathrm{ROHs}(b p)\end{array}$ & $\begin{array}{c}\text { Average sum of length } \\
\text { of ROHs per individual (bp) }\end{array}$ & $\begin{array}{c}\text { Chromosome } \\
\text { size }(b p)^{\mathrm{a}}\end{array}$ & $\begin{array}{c}\text { Proportion (\%) of chromosome } \\
\text { encompassed by } \mathrm{ROHs}\end{array}$ & $\begin{array}{l}\text { Number of SNPS } \\
\text { in Affymetrix } 6.0\end{array}$ & $\begin{array}{c}\text { Density of SNPs } \\
\text { per } 10 \mathrm{~kb}\end{array}$ \\
\hline 1 & 1243 & 14.3 & 1029256231 & 11830531 & 247249719 & 4.78 & 73469 & 3.0 \\
\hline 2 & 1491 & 17.1 & 1223537523 & 14063650 & 242951149 & 5.79 & 75933 & 3.1 \\
\hline 3 & 1256 & 14.4 & 1069972110 & 12298530 & 199501827 & 6.16 & 62316 & 3.1 \\
\hline 4 & 1246 & 14.3 & 1015875656 & 11676732 & 191273063 & 6.10 & 57561 & 3.0 \\
\hline 5 & 1021 & 11.7 & 859950902 & 9884493 & 180857866 & 5.47 & 57967 & 3.2 \\
\hline 6 & 1008 & 11.6 & 834180388 & 9588280 & 170899992 & 5.61 & 57855 & 3.4 \\
\hline 7 & 811 & 9.3 & 632768685 & 7273203 & 158821424 & 4.58 & 48419 & 3.0 \\
\hline 8 & 896 & 10.3 & 762529281 & 8764704 & 146274826 & 5.99 & 50019 & 3.4 \\
\hline 9 & 566 & 6.5 & 439197494 & 5048247 & 140273252 & 3.60 & 42710 & 3.0 \\
\hline 10 & 722 & 8.3 & 612229774 & 7037124 & 135374737 & 5.20 & 49608 & 3.7 \\
\hline 11 & 722 & 8.3 & 650352277 & 7475314 & 134452384 & 5.56 & 45944 & 3.4 \\
\hline 12 & 725 & 8.3 & 679233723 & 7807284 & 132349534 & 5.90 & 43833 & 3.3 \\
\hline 13 & 482 & 5.5 & 360268323 & 4141015 & 114142980 & 3.63 & 35158 & 3.1 \\
\hline 14 & 571 & 6.6 & 448210796 & 5151848 & 106368585 & 4.84 & 28942 & 2.7 \\
\hline 15 & 438 & 5.0 & 371570656 & 4270927 & 100338915 & 4.26 & 26905 & 2.7 \\
\hline 16 & 192 & 2.2 & 159973057 & 1838771 & 88827254 & 2.07 & 28658 & 3.2 \\
\hline 17 & 428 & 4.9 & 352288646 & 4049295 & 78774742 & 5.14 & 21347 & 2.7 \\
\hline 18 & 330 & 3.8 & 234464335 & 2694992 & 76117153 & 3.54 & 27219 & 3.6 \\
\hline 19 & 184 & 2.1 & 143788195 & 1652738 & 63811651 & 2.59 & 12419 & 1.9 \\
\hline 20 & 271 & 3.1 & 220116198 & 2530071 & 62435964 & 4.05 & 23487 & 3.8 \\
\hline 21 & 100 & 1.1 & 71684424 & 823959 & 46944323 & 1.76 & 12948 & 2.8 \\
\hline 22 & 112 & 1.3 & 100622242 & 1156577 & 49691432 & 2.33 & 12059 & 2.4 \\
\hline
\end{tabular}

Abbreviations: ROHs, regions of homozygosity; SNPs, single-nucleotide polymorphisms; UCSC, University of California Santa Cruz genes. aThe size of chromosome was obtained from UCSC Genome Browser.

A full colour version of this Table is available at the Journal of Human Genetics Journal online.

we analyzed the CNP output for the HapMap III populations generated by Canary similar to the Swedish population output. As expected, the results of our analysis showed that the CNP profile of the Swedish population was substantially different from that of the African populations (ASW, MKK and LWK) and CHD. More interestingly, the CNP profile of the Swedish population was also considerably different from that of other European populations (MEX and TSI) and GIH. This further supports the importance of delineating the population characteristics of CNVs/CNPs in the Swedish population.

There are a number of limitations when using SNP genotyping arrays to detect $\mathrm{CNV}$ s and $\mathrm{ROH}$, and the $\mathrm{CNV}$ and $\mathrm{ROH}$ list reported in our study is not complete. Future studies will require higher sensitivity methods and larger sample sizes for a more thorough detection of CNVs and ROHs. Nevertheless, this is the first population-based study to investigate the population characteristics of CNVs and ROHs in the Swedish population. This study found many reliable $\mathrm{CNV}$ loci and also highlighted numerous loci that warrant further investigation for their medical or pharmacogenetic importance. The abundance of ROHs detected in the human genome also suggests the importance of studying their associations with complex phenotypes.

\section{ACKNOWLEDGEMENTS}

The Yong Loo Lin School of Medicine, the Life Science Institute and the Office of Deputy President (Research and Technology), National University of Singapore. We also acknowledge the support of the Genome Institute of Singapore, and Agency for Science, Technology and Research, Singapore.

1 McCarroll, S. A., Kuruvilla, F. G., Korn, J. M., Cawley, S., Nemesh, J., Wysoker, A. et al. Integrated detection and population-genetic analysis of SNPs and copy number variation. Nat. Genet. 40, 1166-1174 (2008).

2 Conrad, D. F., Pinto, D., Redon, R., Feuk, L., Gokcumen, O., Zhang, Y. et al. Origins and functional impact of copy number variation in the human genome. Nature 464, 704-712 (2010).
3 Park, H., Kim, J. I., Ju, Y. S., Gokcumen, O., Mills, R. E., Kim, S. et al. Discovery of common Asian copy number variants using integrated high-resolution array $\mathrm{CGH}$ and massively parallel DNA sequencing. Nat. Genet. 42, 400-405 (2010).

4 Yim, S. H., Kim, T. M., Hu, H. J., Kim, J. H., Kim, B. J., Lee, J. Y. et al. Copy number variations in East-Asian population and their evolutionary and functional implications. Hum. Mol. Genet. 19, 1001-1008 (2010).

5 Ku, C. S., Pawitan, Y., Sim, X., Ong, R. T., Seielstad, M., Lee, E. J. et al. Genomic copy number variations in three Southeast Asian populations. Hum. Mutat. 31, 851-857 (2010).

6 Redon, R., Ishikawa, S., Fitch, K. R., Feuk, L., Perry, G. H., Andrews, T. D. et al. Global variation in copy number in the human genome. Nature 444, 444-454 (2006).

7 Pinto, D., Marshall, C., Feuk, L. \& Scherer, S. W. Copy-number variation in control population cohorts. Hum. Mol. Genet. 16, R168-R173 (2007).

8 Zogopoulos, G., Ha, K. C., Naqib, F., Moore, S., Kim, H., Montpetit, A. et al. Germ-line DNA copy number variation frequencies in a large North American population. Hum. Genet. 122, 345-353 (2007).

9 de Smith, A. J., Tsalenko, A., Sampas, N., Scheffer, A., Yamada, N. A., Tsang, P. et al. Array CGH analysis of copy number variation identifies 1284 new genes variant in healthy white males: implications for association studies of complex diseases. Hum. Mol. Genet. 16, 2783-2794 (2007).

10 Díaz de Ståhl, T., Sandgren, J., Piotrowski, A., Nord, H., Andersson, R., Menzel, U. et al. Profiling of copy number variations (CNVs) in healthy individuals from three ethnic groups using a human genome $32 \mathrm{~K}$ BAC-clone-based array. Hum. Mutat. 29, 398-408 (2008).

11 Estivill, X. \& Armengol, L. Copy number variants and common disorders: filling the gaps and exploring complexity in genome-wide association studies. PLoS Genet. 3, 1787-1799 (2007).

12 Gibson, J., Morton, N. E. \& Collins, A. Extended tracts of homozygosity in outbred human populations. Hum. Mol. Genet. 15, 789-795 (2006).

13 Li, L. H., Ho, S. F., Chen, C. H., Wei, C. Y., Wong, W. C., Li, L. Y. et al. Long contiguous stretches of homozygosity in the human genome. Hum. Mutat. 27, 1115-1121 (2006).

14 McQuillan, R., Leutenegger, A. L., Abdel-Rahman, R., Abdel-Rahman, R., Franklin, C. S., Pericic, M. et al. Runs of homozygosity in European populations. Am. J. Hum. Genet. 83, 359-372 (2008).

15 Nothnagel, M., Lu, T. T., Kayser, M. \& Krawczak, M. Genomic and geographic distribution of SNP-defined runs of homozygosity in Europeans. Hum. Mol. Genet. 19, 2927-2935 (2010).

16 Lencz, T., Lambert, C., DeRosse, P., Burdick, K. E., Morgan, T. V., Kane, J. M. et al. Runs of homozygosity reveal highly penetrant recessive loci in schizophrenia. Proc. Natl Acad. Sci. USA 104, 19942-19947 (2007).

17 Nalls, M. A., Guerreiro, R. J., Simon-Sanchez, J., Bras, J. T., Traynor, B. J., Gibbs, J. R. et al. Extended tracts of homozygosity identify novel candidate genes associated with late-onset Alzheimer's disease. Neurogenetics 10, 183-190 (2009).

18 Yang, T. L., Guo, Y., Zhang, L. S., Tian, Q., Yan, H., Papasian, C. J. et al. Runs of homozygosity identify a recessive locus $12 \mathrm{q} 21.31$ for human adult height. J. Clin. Endocrinol. Metab. 95, 3777-3782 (2010). 
19 O’Dushlaine, C. T., Morris, D., Moskvina, V., Kirov, G., Consortium, I. S., Gill, M. et al. Population structure and genome-wide patterns of variation in Ireland and Britain. Eur. J. Hum. Genet. 18, 1248-1254 (2010).

20 International HapMap 3 Consortium. Integrating common and rare genetic variation in diverse human populations. Nature 467, 52-58 (2010).

21 Wang, K., Li, M., Hadley, D., Liu, R., Glessner, J., Grant, S. F. et al. PennCNV: an integrated hidden Markov model designed for high-resolution copy number variation detection in whole-genome SNP genotyping data. Genome Res. 17, 1665-1674 (2007).

22 Korn, J. M., Kuruvilla, F. G., McCarroll, S. A., Wysoker, A., Nemesh, J., Cawley, S. et al. Integrated genotype calling and association analysis of SNPs, common copy number polymorphisms and rare CNVs. Nat. Genet. 40, 1253-1260 (2008).

23 Mei, T. S., Salim, A., Calza, S., Seng, K. C., Seng, C. K. \& Pawitan, Y. Identification of recurrent regions of copy-number variants across multiple individuals. BMC Bioinformatics 11, 147 (2010).

24 lafrate, A. J., Feuk, L., Rivera, M. N., Listewnik, M. L., Donahoe, P. K., Qi, Y. et al. Detection of large-scale variation in the human genome. Nat. Genet. 36, 949-951 (2004).

25 Aqeilan, R. I., Kuroki, T., Pekarsky, Y., Albagha, O., Trapasso, F., Baffa, Re et al. Loss of WWOX expression in gastric carcinoma. Clin. Cancer Res. 10, 3053-3058 (2004).

26 Kuroki, T., Yendamuri, S., Trapasso, F., Matsuyama, A., Aqeilan, R. I., Alder, H. et al. The tumor suppressor gene WWOX at FRA16D is involved in pancreatic carcinogenesis. Clin. Cancer Res. 10, 2459-2465 (2004).

27 Prickett, T. D., Agrawal, N. S., Wei, X., Yates, K. E., Lin, J. C., Wunderlich, J. R. et al. Analysis of the tyrosine kinome in melanoma reveals recurrent mutations in ERBB4. Nat. Genet. 41, 1127-1132 (2009).

28 Ferreira, M. A., O'Donovan, M. C., Meng, Y. A., Jones, I. R., Ruderfer, D. M., Jones, L. et al. Collaborative genome-wide association analysis supports a role for ANK3 and CACNA1C in bipolar disorder. Nat. Genet. 40, 1056-1058 (2008).

29 Ouahchi, K., Lindeman, N. \& Lee, C. Copy number variants and pharmacogenomics. Pharmacogenomics 7, 25-29 (2006).

30 Fanciulli, M., Norsworthy, P. J., Petretto, E., Dong, R., Harper, L., Kamesh, L. et al. FCGR3B copy number variation is associated with susceptibility to systemic, but not organ-specific, autoimmunity. Nat. Genet. 39, 721-723 (2007).
31 Miki, D., Kubo, M., Takahashi, A., Yoon, K. A., Kim, J., Lee, G. K. et al. Variation in TP63 is associated with lung adenocarcinoma susceptibility in Japanese and Korean populations. Nat. Genet. 42, 893-896 (2010).

32 Spencer, K. L., Hauser, M. A., Olson, L. M., Schmidt, S., Scott, W. K., Gallins, P. et al. Deletion of CFHR3 and CFHR1 genes in age-related macular degeneration. Hum. Mol. Genet. 17, 971-977 (2008).

33 Karypidis, A. H., Olsson, M., Andersson, S. O., Rane, A. \& Ekström, L. Deletion polymorphism of the UGT2B17 gene is associated with increased risk for prostate cancer and correlated to gene expression in the prostate. Pharmacogenomics J. 8, 147-151 (2008).

34 McCarroll, S. A., Bradner, J. E., Turpeinen, H., Volin, L., Martin, P. J., Chilewski, S. D. et al. Donor-recipient mismatch for common gene deletion polymorphisms in graftversus-host disease. Nat. Genet. 41, 1341-1344 (2009).

35 Docampo, E., Rabionet, R., Riveira-Muñoz, E., Escaramís, G., Julià, A., Marsal, S. et al. Deletion of the late cornified envelope genes, LCE3C and LCE3B, is associated with rheumatoid arthritis. Arthritis Rheum. 62, 1246-1251 (2010).

36 de Cid, R., Riveira-Munoz, E., Zeeuwen, P. L., Robarge, J., Liao, W., Dannhauser, E. N. et al. Deletion of the late cornified envelope LCE3B and LCE3C genes as a susceptibility factor for psoriasis. Nat. Genet. 41, 211-215 (2009).

37 Wang, J., Wang, W., Li, R., Li, Y., Tian, G., Goodman, L. et al. The diploid genome sequence of an Asian individual. Nature 456, 60-65 (2008).

38 Wheeler, D. A., Srinivasan, M., Egholm, M., Shen, Y., Chen, L., McGuire, A. et al. The complete genome of an individual by massively parallel DNA sequencing. Nature 452 , 872-876 (2008).

39 Korbel, J. O., Urban, A. E., Affourtit, J. P., Godwin, B., Grubert, F., Simons, J. F. et al. Paired-end mapping reveals extensive structural variation in the human genome. Science 318, 420-426 (2007).

40 Willer, C. J., Speliotes, E. K., Loos, R. J., Li, S., Lindgren, C. M., Heid, I. M. et al. Six new loci associated with body mass index highlight a neuronal influence on body weight regulation. Nat. Genet. 41, 25-34 (2009).

41 McCarroll, S. A., Huett, A., Kuballa, P., Chilewski, S. D., Landry, A., Goyette, P. et al. Deletion polymorphism upstream of IRGM associated with altered IRGM expression and Crohn's disease. Nat. Genet. 40, 1107-1112 (2008).

Supplementary Information accompanies the paper on Journal of Human Genetics website (http://www.nature.com/jhg) 